\title{
Photoproduction of doubly heavy baryon at the ILC
}

\author{
Gu Chen, Xing-Gang Wu, Zhan Sun, Yang Ma and Hai-Bing Fu \\ Department of Physics, Chongqing University, \\ Chongqing 401331, P.R. China \\ E-mail: cgu@cqu.edu.cn, wuxg@cqu.edu.cn, zhansun@cqu.edu.cn, \\ mayangluon@cqu.edu.cn, fuhb@cqu.edu.cn
}

ABSTRACT: In the present paper, we make a detailed study on the doubly heavy baryon photoproduction in the future $e^{+} e^{-}$International Linear Collider (ILC). The baryons $\Xi_{c c}$, $\Xi_{b c}$, and $\Xi_{b b}$ are produced via the channel $\gamma \gamma \rightarrow \Xi_{Q Q^{\prime}}+\bar{Q}^{\prime}+\bar{Q}$, where $Q$ and $Q^{\prime}$ stand for heavy $c$ or $b$ quark, respectively. As for the $\Xi_{Q Q^{\prime}}$-baryon production, it shall first generate a $\left(Q Q^{\prime}\right)[n]$-diquark and then form the final baryon via fragmentation, where $[n]$ stands for the color- and spin- configurations for the $\left(Q Q^{\prime}\right)$-diquark states. According to the nonrelativistic QCD theory, four diquark configurations shall provide sizable contributions to the baryon production, e.g., $[n]$ equals $\left[{ }^{3} S_{1}\right]_{\overline{\mathbf{3}}},\left[{ }^{1} S_{0}\right]_{\mathbf{6}}$, $\left[{ }^{3} S_{1}\right]_{\mathbf{6}}$, or $\left[{ }^{1} S_{0}\right]_{\overline{\mathbf{3}}}$, respectively. We adopt the improved helicity amplitude approach for the hard scattering amplitude to improve the calculation efficiency. Total and differential cross sections of those channels, as well as the theoretical uncertainties, are presented. We show that sizable amounts of baryon events can be generated at the ILC, i.e., about $2.0 \times 10^{6} \Xi_{c c}, 2.2 \times 10^{5} \Xi_{b c}$, as well as $3.0 \times 10^{3} \Xi_{b b}$ events are to be generated in one operation year for $\sqrt{S}=500 \mathrm{GeV}$ and $\mathcal{L} \simeq 10^{36} \mathrm{~cm}^{-2} \mathrm{~s}^{-1}$.

KEywords: QCD Phenomenology, Phenomenological Models

ARXIV EPRINT: 1408.4615 


\section{Contents}

1 Introduction 1

2 Calculation technology 2

3 Numerical results and discussions 5

$\begin{array}{lll}4 & \text { Summary } & 11\end{array}$

\section{Introduction}

Many theoretical predictions for the production of doubly heavy baryons $\Xi_{Q Q^{\prime}}$ have been done in refs. [1-14], where $Q^{(\prime)}$ stands for heavy $b$ or $c$ quark, respectively. For convenience, throughout the paper, we take $\Xi_{Q Q^{\prime}}$ as a short notation for the baryon $\Xi_{Q Q^{\prime} q}$, with $q$ equals to the light quark $u, d$, or $s$, respectively. Among the doubly heavy baryons $\Xi_{c c}, \Xi_{b c}$, and $\Xi_{b b}$, only $\Xi_{c c}$ has been observed by the SELEX fixed-target experiment $[15,16]$. However, the SELEX measurements on the $\Xi_{c c}$ properties, such as its decay width and production rate, are much larger than the theoretical predictions [15-17], even by including the extrinsic and intrinsic charm production mechanisms [7-10]. At present, its observations are also lack of supports from other experiments [18-20]. Thus, in addition to the hadronic platforms, it is helpful to find other platforms which can generate large amounts of baryon events to study the baryon properties more precisely.

We shall study the doubly heavy baryon photoproduction in the future $e^{+} e^{-}$International Linear Collider (ILC) within the framework of the non-relativistic QCD (NRQCD) theory [21]. Within this platform, the doubly heavy baryons can be produced through the channel via a single virtual photon or a $Z^{0}$ boson, $e^{+} e^{-} \rightarrow \gamma^{*} / Z^{0} \rightarrow \Xi_{Q Q^{\prime}}+\bar{Q}^{\prime}+\bar{Q}$, or through the photonproduction channel via the double photon collision, $\gamma \gamma \rightarrow \Xi_{Q Q^{\prime}}+\bar{Q}^{\prime}+\bar{Q}$. It is found that the production cross section for the single photon $/ Z^{0}$ process shall be highly suppressed [13, 14], which is about two orders lower than that of the photoproduction channel. So, in the present paper, we shall concentrate on the photoproduction channel.

One can treat the photoproduction of the baryon by two steps. The first step is for the incident photons coming from the electron and positron beams to produce the heavy $Q \bar{Q}$ and $Q^{\prime} \bar{Q}^{\prime}$ pairs. This step is pQCD calculable and can be treated by using the improved helicity amplitude approach [22]. The second step is that the heavy quarks $Q$ and $Q^{\prime}$ evolving into a binding diquark $\left(Q Q^{\prime}\right)$ with color- and spin- configuration $[n]$, e.g., $[n]=\left[{ }^{3} S_{1}\right] \overline{\mathbf{3}},\left[{ }^{1} S_{0}\right]_{\mathbf{6}}$ for $(c c)$ or $(b b)$ diquark and $(b c)_{\overline{\mathbf{3}}}\left[{ }^{3} S_{1}\right],(b c)_{\mathbf{6}}\left[{ }^{1} S_{0}\right],(b c)_{\overline{\mathbf{3}}}\left[{ }^{1} S_{0}\right]$, and $(b c)_{6}\left[{ }^{3} S_{1}\right]$, respectively. Then, the diquark $\left(Q Q^{\prime}\right)[n]$ shall be hadronized into the doubly heavy baryons $\Xi_{c c}, \Xi_{b c}$, and $\Xi_{b b}$ via fragmentation. Similar factorization procedures have also been suggested for dealing with the $\Lambda_{c}$ or $\Lambda_{b}$ baryon production [23, 24]. 
According to NRQCD, the $\Xi_{Q Q^{\prime}}$ baryon can be expanded over the Fock states,

$$
\left|\Xi_{Q Q^{\prime}}\right\rangle=c_{1}(v)\left|\left(Q Q^{\prime}\right) q\right\rangle+c_{2}(v)\left|\left(Q Q^{\prime}\right) q g\right\rangle+c_{3}(v)\left|\left(Q Q^{\prime}\right) q g g\right\rangle+\cdots,
$$

where $v$ is the relative velocity of the constituent heavy quarks in the baryon rest frame. Usually, it is stated that all the baryons are dominated by the first Fock state $\left|\left(Q Q^{\prime}\right) q\right\rangle$, then the emitted gluon from the heavy quark for $\left(Q Q^{\prime}\right)$ in $\left[{ }^{1} S_{0}\right]_{6}$ state must change the spin of the heavy quark; thus, the probability coefficient $c_{1}(v)$ shall dominant over other coefficients, or equivalently, $h_{\mathbf{6}}$ shall be at least $v^{2}$-suppressed to $h_{\overline{\mathbf{3}}}$ and can be neglected. Here $h_{\overline{\mathbf{3}}}$ stands for the probability of transforming the color antitriplet diquark into the baryon and $h_{6}$ stands for the probability of transforming the color sextuplet diquark into the baryon.

A different power counting rule over $v$-expansion has also been suggested in the literature. Ref. [5] suggests that the second Fock state $\left|\left(Q Q^{\prime}\right) q g\right\rangle$ can be of the same importance as $\left|\left(Q Q^{\prime}\right) q\right\rangle$ [5]. Its main idea lies in that one of the heavy quarks can emit a gluon, which does not need to change the spin of the heavy quark, and this gluon can further split into a light $q \bar{q}$ pair; the light quarks can also emit gluons, and finally, the baryon components can be formed with a light quark $q$ plus one or more soft gluons. Since the light quark can emit gluons easily, we have $c_{1}(v) \sim c_{2}(v) \sim c_{3}(v)$. As a rough order estimation, we take the transition probabilities for those diquark states to form the corresponding baryon to be the same, i.e., $h_{\mathbf{6}} \simeq h_{\overline{\mathbf{3}}}$. Following this approximation, we shall find that the color sextuplet diquark component can also provide sizable contributions to the baryon production. It is found that those matrix elements are overall parameters, and their uncertainties can be conveniently discussed when we know their values well. For convenience, we will adopt the assumption $h_{\mathbf{6}} \simeq h_{\overline{\mathbf{3}}}$ to do our discussions throughout the paper.

The remaining parts of the paper are organized as follows. In section 2 , we present the formulation for dealing with the photoproduction channel $\gamma \gamma \rightarrow \Xi_{Q Q^{\prime}}+\bar{Q}^{\prime}+\bar{Q}$ at the leading-order level. In section 3, we give the numerical results. Section 4 is reserved for a summary.

\section{Calculation technology}

At the leading order $\mathcal{O}\left(\alpha^{2} \alpha_{s}^{2}\right)$, within the NRQCD factorization approach, the differential cross section for the channel $\gamma \gamma \rightarrow \Xi_{Q Q^{\prime}}+\bar{Q}^{\prime}+\bar{Q}$ can be formulated as

$$
d \sigma=\int d x_{1} d x_{2} f_{\gamma}\left(x_{1}\right) f_{\gamma}\left(x_{2}\right) \int d z D_{Q Q^{\prime}}^{H}(z) \times \frac{1}{2 x_{1} x_{2} S} \bar{\sum}|\mathcal{M}|^{2} d \Phi_{3}\left\langle\mathcal{O}^{H}(n)\right\rangle,
$$

where $\left\langle\mathcal{O}^{H}(n)\right\rangle$ stands for the long-distance matrix element, which is proportional to the inclusive transition probability of the perturbative state, $\left(Q Q^{\prime}\right)[n]$ pair into the heavy baryon $\Xi_{Q Q^{\prime}} \cdot \mathcal{M}$ is the hard scattering amplitude, which is calculable since the intermediate gluon should be hard enough to generate a heavy $Q \bar{Q}$ or $Q^{\prime} \bar{Q}^{\prime}$ pair. $\bar{\sum}$ means we need to average over the spin states of the electron and positron and sum over the color and spin of all final particles. $d \Phi_{3}$ is the conventional three-body phase space. $f_{\gamma}(x)$ is the density function of the incident photon $[25,26]$. 


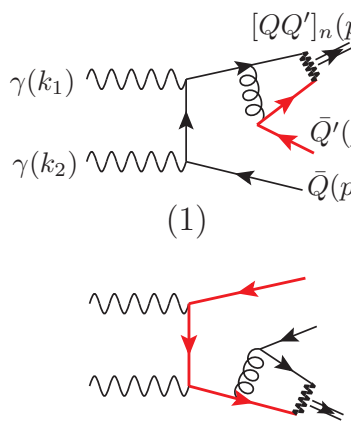

(6)

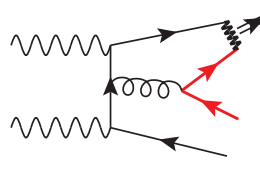

(2)

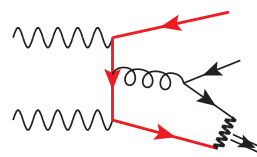

(7)

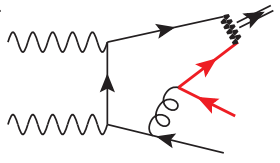

(3)

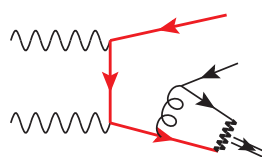

(8)

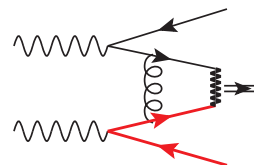

(4)

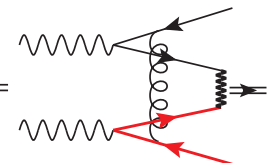

(5)

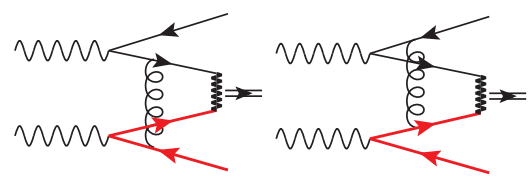

(9)

Figure 1. Typical Feynman diagrams for the production channel $\gamma\left(k_{1}\right)+\gamma\left(k_{2}\right) \rightarrow \Xi_{Q Q^{\prime}}\left(p_{3}\right)+$ $\bar{Q}^{\prime}\left(p_{4}\right)+\bar{Q}\left(p_{5}\right)$ at the tree level. Other ten diagrams can be obtained by exchanging the positions of the initial photons attached to the quark lines.

There are totally 20 Feynman diagrams for the channel $\gamma\left(k_{1}\right)+\gamma\left(k_{2}\right) \rightarrow \Xi_{Q Q^{\prime}}\left(p_{3}\right)+$ $\bar{Q}^{\prime}\left(p_{4}\right)+\bar{Q}\left(p_{5}\right)$. We put 10 diagrams in figure 1 , the other 10 Feynman diagrams can be obtained by exchanging the positions of the initial photons attached to the quark lines. The fragmentation function $D_{Q Q^{\prime}}^{H}$ can be estimated within certain phenomenological models [27-38]. We adopt the following form suggested by ref. [27] for our calculation

$$
D_{Q Q^{\prime}}^{H}(z)=\frac{N_{Q Q^{\prime}}}{z\left[1-(1 / z)-\epsilon_{Q Q^{\prime}} /(1-z)\right]^{2}},
$$

where $\epsilon_{c c}=\left(m_{q} / M_{\Xi_{c c}}\right)^{2} \epsilon_{b}, \epsilon_{b c}=\left(m_{q} / M_{\Xi_{b c}}\right)^{2} \epsilon_{b}$, and $\epsilon_{b b}=\left(m_{q} / M_{\Xi_{b b}}\right)^{2} \epsilon_{b}$. The light quark mass is chosen as $m_{q}=0.3 \mathrm{GeV}$ [39]. The parameter $\epsilon_{b}$ can be fixed by comparing with the data [40-44], which is $\sim 0.004$ [45]. The normalization factor $N_{Q Q^{\prime}}$ can be fixed by the normalization condition, $\int D_{Q Q^{\prime}}^{H}(z) d z=1$, which leads to $N_{c c}=0.0084, N_{b c}=0.00386$, and $N_{b b}=0.0025$ for $\Xi_{c c}, \Xi_{b c}$, and $\Xi_{b b}$, respectively.

The hard scattering amplitude $\mathcal{M}=\sum_{k=1}^{20} \mathcal{M}_{k}$ for the process can be written in a general form as

$$
\begin{aligned}
\mathcal{M}_{k}= & \mathcal{C}_{i j l} \times X_{k} \times \sum_{s_{1}, s_{3}} \bar{u}_{s_{1}}\left(p_{32}\right) \Gamma_{1} s_{f}\left(k_{1}, m_{Q^{\prime}}\right) \cdots s_{f}\left(k_{\rho-1}, m_{Q^{\prime}}\right) \Gamma_{\rho} v_{s_{2}}\left(p_{4}\right) \\
& \times \bar{u}_{s_{3}}\left(p_{31}\right) \Gamma_{1}^{\prime} s_{f}\left(k_{1}^{\prime}, m_{Q}\right) \cdots s_{f}\left(k_{\kappa-1}^{\prime}, m_{Q}\right) \Gamma_{\kappa}^{\prime} v_{s_{4}}\left(p_{5}\right),
\end{aligned}
$$

where $k=(1, \ldots, 20), \Gamma_{1}, \ldots, \Gamma_{\rho}$ and $\Gamma_{1}^{\prime}, \ldots, \Gamma_{\kappa}^{\prime}$ are interaction vertexes, which contain the Dirac- $\gamma$ matrixes only. $s_{f}\left(k_{1}^{(\prime)}, m_{Q^{(\prime)}}\right)$ and the like are fermion propagators. $X_{k}$ is the scalar part of the propagators for the whole amplitude. The momentum of the constituent quarks are $p_{31}=\frac{m_{Q}}{M_{Q Q^{\prime}}} p_{3}$ and $p_{32}=\frac{m_{Q^{\prime}}}{M_{Q Q^{\prime}}} p_{3} . \mathcal{C}_{i j l}$ is the color factor defined as

$$
\mathcal{C}_{i j l}=\mathcal{N}_{c} \times \sum_{m, n}\left(T^{a}\right)_{i m}\left(T^{a}\right)_{j n} \times G_{m n l}
$$

where the subindices $m$ and $n$ are color indices of the constituent heavy quarks, and $l$ is the color of the diquark. $a=1, \ldots, 8$ is the color index of the gluon propagator. $\mathcal{N}_{c}=1 / \sqrt{2}$ is 
the normalization factor. The function $G_{m n l}$ equals the antisymmetric $\varepsilon_{m n l}$ (the symmetric $\left.f_{m n l}\right)$ for the color antitriplet $\overline{\mathbf{3}}$ (the color sextuplet $\left.\mathbf{6}\right)$ of $\left(Q Q^{\prime}\right)$ diquark. The sum of the anti-symmetric and the symmetric functions satisfy the following equations

$$
\varepsilon_{m n l} \varepsilon_{m^{\prime} n^{\prime} l}=\delta_{m m^{\prime}} \delta_{n n^{\prime}}-\delta_{m n^{\prime}} \delta_{n m^{\prime}}
$$

and

$$
f_{m n l} f_{m^{\prime} n^{\prime} l}=\delta_{m m^{\prime}} \delta_{n n^{\prime}}+\delta_{m n^{\prime}} \delta_{n m^{\prime}}
$$

With the help of the above relations, we obtain $\mathcal{C}_{i j l}^{2}=\frac{4}{3}$ for the color-antitriplet diquark state and $\mathcal{C}_{i j l}^{2}=\frac{2}{3}$ for the color-sextuplet diquark state, respectively.

All the amplitudes $\mathcal{M}_{k}$ with $k=(1, \ldots, 20)$ contain massive quark lines, so it is too complicated and lengthy by using the conventional trace technique to deal with the amplitude square. To shorten the calculations and to make the results more compact, we adopt the improved helicity amplitude approach [22] to deal with the difficulty of calculating the expressions for the yields when the quark masses cannot be neglected. It is found that we can connect the doubly heavy baryon production with those of doubly heavy quarkonium production. We have made a detailed discussion on the heavy quarkonium production at the ILC under the improved helicity amplitude approach via the channel $\gamma \gamma \rightarrow\left|\left[Q \bar{Q}^{\prime}\right](n)\right\rangle+Q^{\prime}+\bar{Q}$ in ref. [46]. To compare with the quarkonium case, by applying the charge conjugation matrix $C=-i \gamma^{2} \gamma^{0}$ and the transverse of the matrix element to the amplitude $\mathcal{M}_{k}$, we can transform eq. (2.3) as

$$
\begin{aligned}
\mathcal{M}_{k}= & (-1)^{\rho+1} \mathcal{C}_{i j} \times X_{k} \times \sum_{s_{1}, s_{3}} \bar{u}_{s_{2}}\left(p_{4}\right) \Gamma_{\rho} s_{f}\left(-k_{\rho-1}, m_{Q^{\prime}}\right) \cdots s_{f}\left(-k_{1}, m_{Q^{\prime}}\right) \Gamma_{1} v_{s_{1}}\left(p_{32}\right) \\
& \times \bar{u}_{s_{3}}\left(p_{31}\right) \Gamma_{1}^{\prime} s_{f}\left(k_{1}^{\prime}, m_{Q}\right) \cdots s_{f}\left(k_{\kappa-1}^{\prime}, m_{Q}\right) \Gamma_{\kappa}^{\prime} v_{s_{4}}\left(p_{5}\right) \\
= & (-1)^{\rho+1} \mathcal{C}_{i j} X_{k} \bar{u}_{s_{2}}\left(p_{4}\right) \Gamma_{\rho} s_{f}\left(-k_{\rho-1}, m_{Q^{\prime}}\right) \cdots s_{f}\left(-k_{1}, m_{Q^{\prime}}\right) \Pi\left(p_{3}\right) \\
& \times \Gamma_{1}^{\prime} s_{f}\left(k_{1}^{\prime}, m_{Q}\right) \cdots s_{f}\left(k_{\kappa-1}^{\prime}, m_{Q}\right) \Gamma_{\kappa}^{\prime} v_{s_{4}}\left(p_{5}\right)
\end{aligned}
$$

where $\rho$ stands for the number of the $\gamma$-matrixes appearing in the amplitude $\mathcal{M}_{k}$. The second line is the matrix element for the heavy quarkonium production, which indicates that the amplitudes for the diquark production are merely different from those of the heavy quarkonium case with an overall factor $(-1)^{\rho+1}$. Thus, inversely, we can conveniently derive the hard scattering amplitudes $\mathcal{M}_{k}$ from ref. [46] after proper transformation. To shorten the paper, we will not put the detailed calculation technology for the baryon production here, the interesting readers may turn to ref. [46] for details of the improved helicity amplitude approach. Here, to derive eq. (2.7), we have implicitly applied the relations: $C C^{-1}=1$ and

$$
\begin{aligned}
v_{s}^{T}(p) C & =-\bar{u}_{s}(p), & C^{-1} \bar{u}_{s}^{T}(p) & =v_{s}(p), \\
C^{-1} s_{f}^{T}\left(k_{1}, m_{Q}\right) C & =s_{f}\left(-k_{1}, m_{Q}\right), & C^{-1} \Gamma_{n}^{T} C & =-\Gamma_{n} .
\end{aligned}
$$




\begin{tabular}{|c|c|c|c|}
\hline & $250(\mathrm{GeV})$ & $500(\mathrm{GeV})$ & $1(\mathrm{TeV})$ \\
\hline$(c c)_{\mathbf{6}}\left[{ }^{1} S_{0}\right]$ & 39.27 & 18.45 & 7.53 \\
\hline$(c c)_{\overline{\mathbf{3}}}\left[{ }^{3} S_{1}\right]$ & 434.86 & 183.81 & 71.92 \\
\hline$(b c)_{\overline{\mathbf{3}}}\left[{ }^{3} S_{1}\right]$ & 21.81 & 10.45 & 4.48 \\
\hline$(b c)_{\mathbf{6}}\left[{ }^{1} S_{0}\right]$ & 4.81 & 2.27 & 0.96 \\
\hline$(b c)_{\mathbf{6}}\left[{ }^{3} S_{1}\right]$ & 10.91 & 5.22 & 2.24 \\
\hline$(b c)_{\overline{\mathbf{3}}}\left[{ }^{1} S_{0}\right]$ & 9.62 & 4.53 & 1.92 \\
\hline$(b b)_{\mathbf{6}}\left[{ }^{1} S_{0}\right]$ & 0.04 & 0.02 & 0.01 \\
\hline$(b b)_{\overline{\mathbf{3}}}\left[{ }^{3} S_{1}\right]$ & 0.53 & 0.28 & 0.13 \\
\hline
\end{tabular}

Table 1. Total cross sections (in unit:fb) for the photoproduction of $\Xi_{c c}, \Xi_{b c}$, and $\Xi_{b b}$ under various color- and spin- configurations at the ILC.

\section{Numerical results and discussions}

As discussed in the Introduction, we adopt $h_{\mathbf{6}} \simeq h_{\overline{\mathbf{3}}}$ to do our discussion. The nonperturbative matrix element with color antitriplet diquark, $h_{\overline{\mathbf{3}}}$, can be related to the Schrödinger wave functions at the origin $\left|\psi_{\left(Q \bar{Q}^{\prime}\right)}(0)\right|$ as [5]:

$$
h_{\overline{\mathbf{3}}}=\left\langle\mathcal{O}^{H}(1 S)\right\rangle \simeq\left|\psi_{\left|\left(Q \bar{Q}^{\prime}\right)[1 S]\right\rangle}(0)\right|^{2} .
$$

Since the spin-splitting effect is small, we do not distinguish the bound state parameters for the spin-singlet and the spin-triplet states; i.e., those parameters, such as the constituent quark masses, the bound state mass, and the wave function, are taken to be the same for the spin-singlet and spin-triplet states. We take the wavefunctions at the origin as [3]: $\left|\Psi_{c c}(0)\right|^{2}=0.039 \mathrm{GeV}^{3},\left|\Psi_{b c}(0)\right|^{2}=0.065 \mathrm{GeV}^{3}$, and $\left|\Psi_{b b}(0)\right|^{2}=0.152 \mathrm{GeV}^{3}$. The heavy quark masses are taken as: $m_{c}=1.5 \mathrm{GeV}$ and $m_{b}=4.9 \mathrm{GeV}$. The doubly heavy baryon mass is taken as $M_{\Xi_{Q Q^{\prime}}}=m_{Q}+m_{Q^{\prime}}$. The other parameters are taken as the same as those of ref. [46], e.g., the renormalization scale is taken as the transverse mass, $\mu_{r}=M_{t}=$ $\sqrt{M_{Q Q^{\prime}}^{2}+p_{t}^{2}}$. As a cross check of our calculation, we obtain same numerical results as those derived from the conventional squared amplitude approach.

Total cross sections for the doubly heavy baryon photoproduction with three collision energies, i.e., $\sqrt{S}=250 \mathrm{GeV}, 500 \mathrm{GeV}$, and $1 \mathrm{TeV}$, are put in table 1 . Summing up the contributions from different color- and spin- configurations, we find that the total cross sections decrease with the increment of $\sqrt{S}$, i.e.,

$$
\begin{aligned}
& \left.\sigma_{\Xi_{c c}}\right|_{250 \mathrm{GeV}}:\left.\sigma_{\Xi_{c c}}\right|_{500 \mathrm{GeV}}:\left.\sigma_{\Xi_{c c}}\right|_{1 \mathrm{TeV}} \simeq 6: 3: 1, \\
& \left.\sigma_{\Xi_{b c}}\right|_{250 \mathrm{GeV}}:\left.\sigma_{\Xi_{b c}}\right|_{500 \mathrm{GeV}}:\left.\sigma_{\Xi_{b c}}\right|_{1 \mathrm{TeV}} \simeq 5: 2: 1, \\
& \left.\sigma_{\Xi_{b b}}\right|_{250 \mathrm{GeV}}:\left.\sigma_{\Xi_{b b}}\right|_{500 \mathrm{GeV}}:\left.\sigma_{\Xi_{b b}}\right|_{1 \mathrm{TeV}} \simeq 4: 2: 1 .
\end{aligned}
$$

It is noted that the relative importance among different color- and spin- configurations for the total cross sections and the differential distributions are similar under different collision energies. In the following, we take $\sqrt{S}=500 \mathrm{GeV}$ as the $e^{+} e-$ collision energy. 


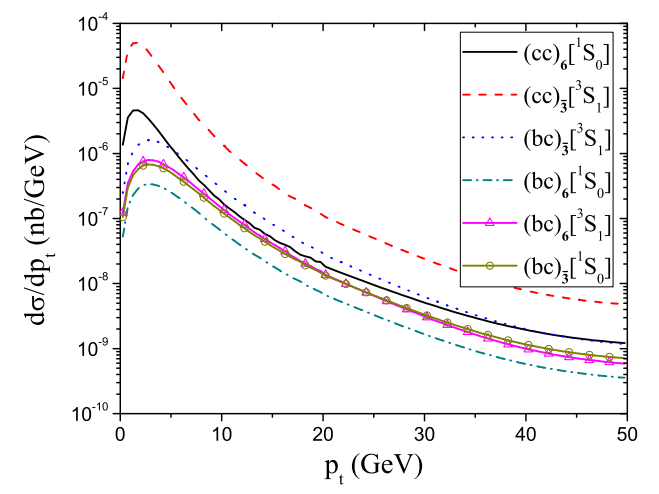

Figure 2. The baryon $p_{t}$ distributions for the process $\gamma \gamma \rightarrow \Xi_{Q Q^{\prime}}+\bar{Q}^{\prime}+\bar{Q}$ at the ILC with $\sqrt{S}=500 \mathrm{GeV}$, where the production via different $\left(Q Q^{\prime}\right)[n]$-diquark configurations are presented.

Under the condition of $\sqrt{S}=500 \mathrm{GeV}$, we obtain

$$
\begin{aligned}
& \sigma_{(c c)_{\overline{3}}\left[{ }^{3} S_{1}\right]}: \sigma_{(c c)_{\mathbf{6}}\left[{ }^{1} S_{0}\right]} \simeq 10: 1, \\
& \sigma_{(b c)_{\overline{3}}\left[{ }^{3} S_{1}\right]}: \sigma_{(b c)_{\mathbf{6}}\left[{ }^{1} S_{0}\right]}: \sigma_{(b c)_{\mathbf{6}}\left[{ }^{3} S_{1}\right]}: \sigma_{(b c)_{\overline{\mathbf{3}}}\left[{ }^{3} S_{1}\right]} \simeq 5: 1: 2: 2, \\
& \sigma_{(b b)_{\overline{3}}\left[{ }^{3} S_{1}\right]}: \sigma_{(b b)_{\mathbf{6}}\left[{ }^{1} S_{0}\right]} \simeq 14: 1 .
\end{aligned}
$$

It indicates that the $\left[{ }^{3} S_{1}\right]_{\overline{3}}$ diquark state provides the dominant contribution, while other configurations may also provide significant contributions. By summing up all the possible diquark configurations, we obtain $\sigma_{\Xi_{c c}}=202.26 \mathrm{fb}, \sigma_{\Xi_{b c}}=22.47 \mathrm{fb}$, and $\sigma_{\Xi_{b b}}=0.3 \mathrm{fb}$. If the integrated luminosity is as high as $10^{4} \mathrm{fb}^{-1}$, we shall have about $2.0 \times 10^{6} \Xi_{c c}, 2.2 \times 10^{5}$ $\Xi_{b c}$, and $3.0 \times 10^{3} \Xi_{b b}$ events to be generated through the direct photon collision at the ILC in an operation year. The $\Xi_{c c}$ production rate is larger than those of $\Xi_{b c}$ and $\Xi_{b b}$, i.e., $\sigma_{\Xi_{c c}}: \sigma_{\Xi_{b c}}: \sigma_{\Xi_{b b}}=647: 75: 1$. Thus, in the following, we shall focus on the photoproduction of $\Xi_{c c}$ and $\Xi_{b c}$.

Figure 2 shows the baryon transverse momentum $\left(p_{t}\right)$ distributions for the photoproduction of $\Xi_{c c}$ and $\Xi_{b c}$. Similar to the above conclusion, the $\left[{ }^{3} S_{1}\right]_{\overline{\mathbf{3}}}$ configuration for both $\Xi_{c c}$ and $\Xi_{b c}$ production provides dominant contributions over the other configurations in the whole $p_{t}$ region. We present the rapidity $(y)$ and pseudorapidity $\left(y_{p}\right)$ distributions in figures 3 and 4 . There is a plateau within $|y|<4$ or $\left|y_{p}\right|<4$. We present the differential cross sections $d \sigma / d z$ in figure 5 , where $z=\frac{2}{\hat{s}}\left(k_{1}+k_{2}\right) \cdot p_{3}$ with $\hat{s}=x_{1} x_{2} S$ being the invariant mass of the initial photons of the subprocess. In the subprocess center-of-mass frame, $z$ is simply twice the fraction of the total energy carried by the baryon and is experimentally observable. To be useful references, we present the total cross sections under various $p_{t}$ or $y$ cuts in tables 2 and 3 .

In the literature, people usually takes a simple assumption by treating the evolution from the diquark to doubly heavy baryon with $100 \%$ probability and with equal importance for all phase-space point; we call it the "direct evolution". In the present paper, we have adopted the fragmentation approach with the help of the fragmentation function (2.2) to deal with such evolution; we call it the "evolution via fragmentation". In table 4, we present a comparison of the total cross sections for the baryon photoproduction at the ILC under 


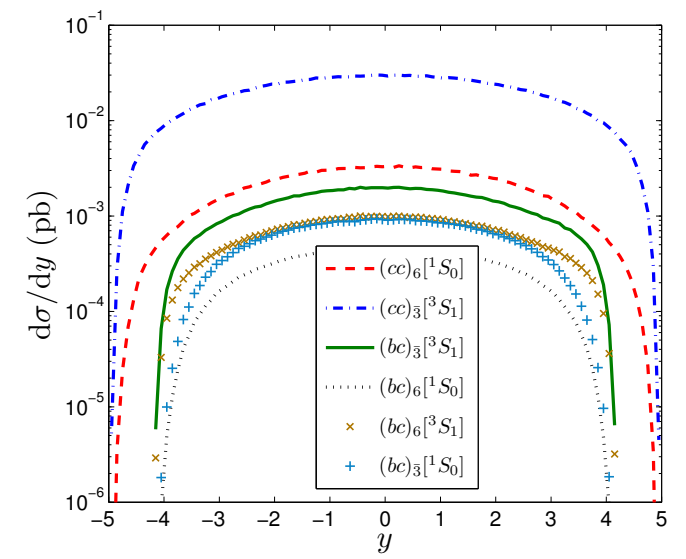

Figure 3. The baryon rapidity distributions for the process $\gamma \gamma \rightarrow \Xi_{Q Q^{\prime}}+\bar{Q}^{\prime}+\bar{Q}$ at the ILC with $\sqrt{S}=500 \mathrm{GeV}$, where the production via different $\left(Q Q^{\prime}\right)[n]$-diquark configurations are presented.

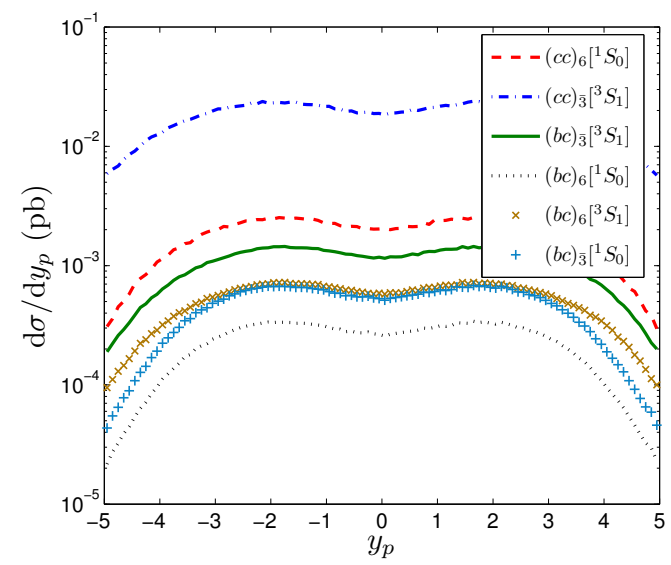

Figure 4. The baryon pseudorapidity distributions for the process $\gamma \gamma \rightarrow \Xi_{Q Q^{\prime}}+\bar{Q}^{\prime}+\bar{Q}$ at the ILC with $\sqrt{S}=500 \mathrm{GeV}$, where the production via different $\left(Q Q^{\prime}\right)[n]$-diquark configurations are presented.

\begin{tabular}{|c|c|c|c|}
\hline$p_{t \text {-cut }}$ & $1 \mathrm{GeV}$ & $2 \mathrm{GeV}$ & $3 \mathrm{GeV}$ \\
\hline$(c c)_{\mathbf{6}}\left[{ }^{1} S_{0}\right]$ & 16.11 & 11.47 & 7.81 \\
\hline$(c c)_{\overline{\mathbf{3}}}\left[{ }^{3} S_{1}\right]$ & 159.24 & 112.44 & 71.25 \\
\hline$(b c)_{\overline{\mathbf{3}}}\left[{ }^{3} S_{1}\right]$ & 10.03 & 8.85 & 7.35 \\
\hline$(b c)_{\mathbf{6}}\left[{ }^{1} S_{0}\right]$ & 2.17 & 1.92 & 1.60 \\
\hline$(b c)_{\mathbf{6}}\left[{ }^{3} S_{1}\right]$ & 5.01 & 4.42 & 3.67 \\
\hline$(b c)_{\overline{\mathbf{3}}}\left[{ }^{1} S_{0}\right]$ & 4.34 & 3.84 & 3.20 \\
\hline
\end{tabular}

Table 2. Total cross sections (in unitsfb) for the photoproduction of $\Xi_{c c}$ and $\Xi_{b c}$ with $\sqrt{S}=$ $500 \mathrm{GeV}$ under various color- and spin- configurations and various $p_{t}$ cuts. 


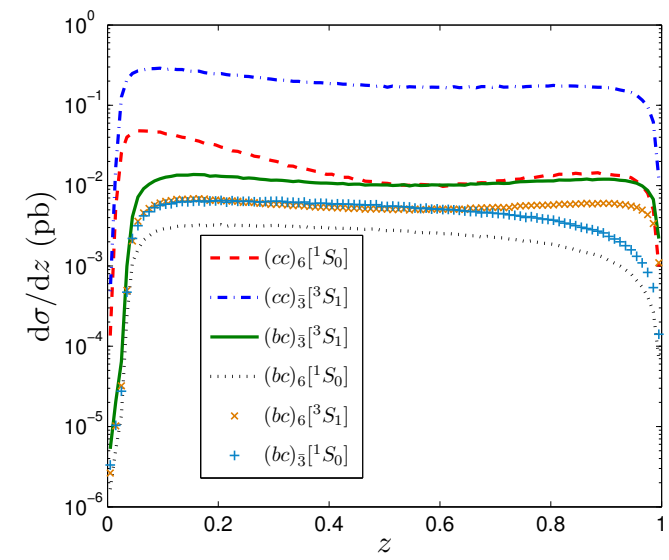

Figure 5. The differential distributions $d \sigma / d z$ for the process $\gamma \gamma \rightarrow \Xi_{Q Q^{\prime}}+\bar{Q}^{\prime}+\bar{Q}$ at the ILC with $\sqrt{S}=500 \mathrm{GeV}$, where the production via different $\left(Q Q^{\prime}\right)[n]$-diquark configurations are presented.

\begin{tabular}{|c|c|c|c|}
\hline$y_{\text {cut }}$ & 1 & 2 & 3 \\
\hline$(c c)_{\mathbf{6}}\left[{ }^{1} S_{0}\right]$ & 5.85 & 11.53 & 15.76 \\
\hline$(c c)_{\overline{\mathbf{3}}}\left[{ }^{3} S_{1}\right]$ & 53.9 & 105.2 & 148.1 \\
\hline$(b c)_{\overline{\mathbf{3}}}\left[{ }^{3} S_{1}\right]$ & 3.76 & 7.02 & 9.35 \\
\hline$(b c)_{\mathbf{6}}\left[{ }^{1} S_{0}\right]$ & 0.85 & 1.61 & 2.12 \\
\hline$(b c)_{\mathbf{6}}\left[{ }^{3} S_{1}\right]$ & 1.88 & 3.51 & 4.67 \\
\hline$(b c)_{\overline{\mathbf{3}}}\left[{ }^{1} S_{0}\right]$ & 1.70 & 3.22 & 4.24 \\
\hline
\end{tabular}

Table 3. Total cross sections (in unitsfb) for the photoproduction of $\Xi_{c c}$ and $\Xi_{b c}$ with $\sqrt{S}=$ $500 \mathrm{GeV}$ under various color- and spin- configurations and various rapidity cuts.

those two treatments. The subscript "d" stands for the "direct evolution", the subscript "f" stands for the "evolution via fragmentation". Table 4 shows the discrepancies of total cross sections for those two treatments are quite small, i.e., less than $\sim 1 \%$. On the other hand, the differences for the $p_{t}$ distributions are also very small in the whole $p_{t}$ region. For example, we put a comparison of the $p_{t}$ distributions under those two treatments in figure 6 . We also present a comparison of the $z$ distributions under those two treatments in figure 7. In those two figures, we have summed up the contributions from the mentioned color- and spin- diquark-configurations for convenience. As for the $\Xi_{c c}$ production, it is found that the differential cross sections for the "evolution via fragmentation" are slightly larger in small $z$ region, while slightly smaller in large $z$ region. This result is consistent with the small differences for the $p_{t}$ distributions under those two treatments. Thus the conventional treatment is viable and provides a good approximation to deal with the heavy baryon production. As a sound estimation, we take the fragmentation approach to do the discussion. 


\begin{tabular}{|c|c|c|}
\hline & $\sigma_{\mathrm{d}}$ & $\sigma_{\mathrm{f}}$ \\
\hline$(c c)_{\mathbf{6}}\left[{ }^{1} S_{0}\right]$ & 18.41 & 18.45 \\
\hline$(c c)_{\overline{\mathbf{3}}}\left[{ }^{3} S_{1}\right]$ & 184.62 & 183.81 \\
\hline$(b c)_{\overline{\mathbf{3}}}\left[{ }^{3} S_{1}\right]$ & 10.49 & 10.45 \\
\hline$(b c)_{\mathbf{6}}\left[{ }^{1} S_{0}\right]$ & 2.28 & 2.27 \\
\hline$(b c)_{\mathbf{6}}\left[{ }^{3} S_{1}\right]$ & 5.24 & 5.22 \\
\hline$(b c)_{\overline{\mathbf{3}}}\left[{ }^{1} S_{0}\right]$ & 4.56 & 4.53 \\
\hline
\end{tabular}

Table 4. Comparison of the total cross sections (in units fb) for the baryon photoproduction at the ILC with $\sqrt{S}=500 \mathrm{GeV}$. The subscript "d" stands for the "direct evolution", the subscript "f" stands for the "evolution via fragmentation".

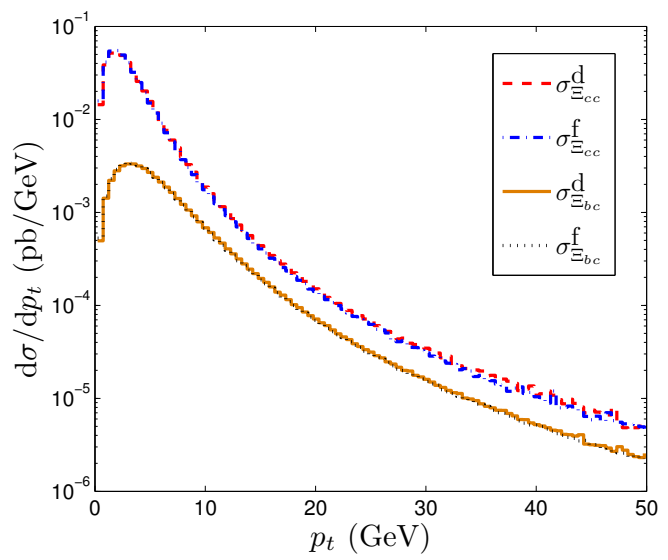

Figure 6. Comparison of the $p_{t}$ distributions for the photoproduction of the $\Xi_{c c}$ and $\Xi_{b c}$ baryons at the ILC with $\sqrt{S}=500 \mathrm{GeV}$. The superscript "d" stands for the "direct evolution", the superscript "f" stands for the "evolution via fragmentation".

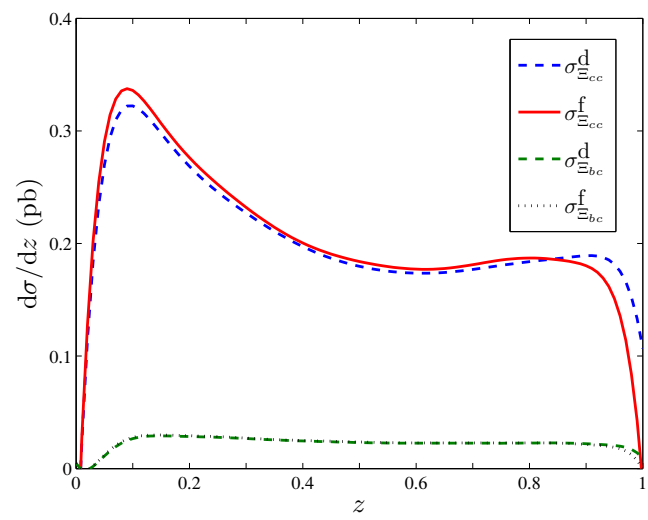

Figure 7. Comparison of the $z$ distributions for the photoproduction of the $\Xi_{c c}$ and $\Xi_{b c}$ baryons at the ILC with $\sqrt{S}=500 \mathrm{GeV}$. The superscript "d" stands for the "direct evolution", the superscript "f" stands for the "evolution via fragmentation". 


\begin{tabular}{|c|c|c|c|}
\hline$m_{c}(\mathrm{GeV})$ & 1.4 & 1.5 & 1.6 \\
\hline$(c c)_{\mathbf{6}}\left[{ }^{1} S_{0}\right]$ & 24.68 & 18.45 & 13.88 \\
\hline$(c c)_{\overline{\mathbf{3}}}\left[{ }^{3} S_{1}\right]$ & 245.38 & 183.81 & 141.30 \\
\hline$(b c)_{\overline{\mathbf{3}}}\left[{ }^{3} S_{1}\right]$ & 12.23 & 10.45 & 9.08 \\
\hline$(b c)_{\mathbf{6}}\left[{ }^{1} S_{0}\right]$ & 2.62 & 2.27 & 1.98 \\
\hline$(b c)_{\mathbf{6}}\left[{ }^{3} S_{1}\right]$ & 6.12 & 5.22 & 4.54 \\
\hline$(b c)_{\overline{\mathbf{3}}}\left[{ }^{1} S_{0}\right]$ & 5.24 & 4.45 & 3.96 \\
\hline
\end{tabular}

Table 5. Uncertainties for the total cross sections (in units fb) by taking $m_{c}=1.5 \pm 0.1 \mathrm{GeV}$. $m_{b}=4.9 \mathrm{GeV}$ and $\mu_{r}=M_{t}$.

\begin{tabular}{|c|c|c|c|}
\hline$m_{b}(\mathrm{GeV})$ & 4.7 & 4.9 & 5.1 \\
\hline$(b c)_{\overline{\mathbf{3}}}\left[{ }^{3} S_{1}\right]$ & 11.37 & 10.45 & 9.67 \\
\hline$(b c)_{\mathbf{6}}\left[{ }^{1} S_{0}\right]$ & 2.46 & 2.27 & 2.07 \\
\hline$(b c)_{\mathbf{6}}\left[{ }^{3} S_{1}\right]$ & 5.68 & 5.22 & 4.83 \\
\hline$(b c)_{\overline{\mathbf{3}}}\left[{ }^{1} S_{0}\right]$ & 4.92 & 4.53 & 4.14 \\
\hline
\end{tabular}

Table 6. Uncertainties for the total cross sections (in unitsfb) by taking $m_{b}=4.9 \pm 0.2 \mathrm{GeV}$. $m_{c}=1.5 \mathrm{GeV}$ and $\mu_{r}=M_{t}$.

As a final remark, we make a discussion on the theoretical uncertainties from the heavy quark masses. For the purpose, we set $m_{c}=1.50 \pm 0.10 \mathrm{GeV}$ and $m_{b}=4.9 \pm 0.20 \mathrm{GeV}$. As shown in table 5 , the uncertainties for $m_{c}=1.50 \pm 0.10 \mathrm{GeV}$ are

$$
\begin{aligned}
\sigma_{(c c)_{\mathbf{6}}\left[{ }^{1} S_{0}\right]} & =18.45_{-4.57}^{+6.23} \mathrm{fb}, \\
\sigma_{(c c)_{\overline{3}}\left[{ }^{3} S_{1}\right]} & =183.81_{-42.51}^{+61.57} \mathrm{fb}, \\
\sigma_{(b c)_{\overline{\mathbf{3}}}\left[{ }^{3} S_{1}\right]} & =10.45_{-1.37}^{+1.78} \mathrm{fb}, \\
\sigma_{(b c)_{\mathbf{6}}\left[{ }^{1} S_{0}\right]} & =2.27_{-0.29}^{+0.35} \mathrm{fb}, \\
\sigma_{(b c)_{\mathbf{6}}\left[{ }^{3} S_{1}\right]} & =5.22_{-0.68}^{+0.89} \mathrm{fb}, \\
\sigma_{(b c)_{\overline{3}}\left[{ }^{1} S_{0}\right]} & =4.53_{-0.58}^{+0.70} \mathrm{fb} .
\end{aligned}
$$

Similarly, as shown in table 6 , the uncertainties caused by the $b$-quark mass $m_{b}=4.9 \pm$ $0.20 \mathrm{GeV}$ are

$$
\begin{aligned}
\sigma_{(b c)_{\overline{3}}\left[{ }^{[} S_{1}\right]} & =10.45_{-0.78}^{+0.92} \mathrm{fb}, \\
\sigma_{(b c)_{\mathbf{6}}\left[{ }^{1} S_{0}\right]} & =2.27_{-0.20}^{+0.19} \mathrm{fb}, \\
\sigma_{(b c)_{\mathbf{6}}\left[{ }^{3} S_{1}\right]} & =5.22_{-0.39}^{+0.46} \mathrm{fb} \\
\sigma_{(b c)_{\overline{3}}\left[{ }^{1} S_{0}\right]} & =4.53_{-0.40}^{+0.38} \mathrm{fb} .
\end{aligned}
$$

We take three scales $\mu_{r}=M_{t}, \sqrt{\hat{s}} / 2$, and $\sqrt{\hat{s}}$ for estimating the scale uncertainties. Numerical results are shown in table 7 , which indicates that the scale uncertainties are $\sim 36 \%$ for $\Xi_{c c}$ and $\sim 26 \%$ for $\Xi_{b c}$. Here we have adopted the improved way as suggested by refs. $[48,49]$ to analyze the scale uncertainty. 


\begin{tabular}{|c|c|c|c|}
\hline$\mu_{r}$ & $\sqrt{\hat{s}}$ & $\sqrt{\hat{s}} / 2$ & $M_{t}$ \\
\hline$(c c)_{\mathbf{6}}\left[{ }^{1} S_{0}\right]$ & 11.29 & 12.87 & 18.45 \\
\hline$(c c)_{\overline{\mathbf{3}}}\left[{ }^{3} S_{1}\right]$ & 117.67 & 135.53 & 183.81 \\
\hline$(b c)_{\overline{\mathbf{3}}}\left[{ }^{3} S_{1}\right]$ & 7.67 & 8.64 & 10.45 \\
\hline$(b c)_{\mathbf{6}}\left[{ }^{1} S_{0}\right]$ & 1.68 & 1.88 & 2.27 \\
\hline$(b c)_{\mathbf{6}}\left[{ }^{3} S_{1}\right]$ & 3.83 & 4.32 & 5.22 \\
\hline$(b c)_{\overline{\mathbf{3}}}\left[{ }^{1} S_{0}\right]$ & 3.36 & 3.76 & 4.53 \\
\hline
\end{tabular}

Table 7. Total cross sections (in units fb) for the heavy quarkonium photoproduction under the improved conventional renormalization scale setting for three scale choices $\mu_{r}=\sqrt{\hat{s}}, \sqrt{\hat{s}} / 2$, and $M_{t}$. $\sqrt{S}=500 \mathrm{GeV}$.

\section{Summary}

We have investigated the photoproduction of the doubly heavy baryons at the ILC within NRQCD. The improved helicity amplitude approach has been adopted to improve the calculation efficiency. By taking the assumption, $h_{6} \simeq h_{\overline{3}}$, we observe that the channel via the intermediate $\left[{ }^{3} S_{1}\right]_{\overline{3}}$ diquark state provides the dominant contribution, while other configurations may also provide significant contributions. Total and differential cross sections, together with their theoretical uncertainties, have been presented. By taking the errors from the heavy quark masses into consideration, we shall have $\left(2.0_{-0.47}^{+0.68}\right) \times 10^{6} \Xi_{c c}$ and $\left(2.2_{-0.29}^{+0.37}\right) \times 10^{5} \Xi_{b c}$ events to be produced in one operation year at the ILC with $\sqrt{S}=500 \mathrm{GeV}$ and $\mathcal{L} \simeq 10^{36} \mathrm{~cm}^{-2} \mathrm{~s}^{-1}$. Thus, the ILC would provide another good platform for studying $\Xi_{Q Q^{\prime}}$-baryon properties.

As a final remark, we discuss the possibility of distinguishing the baryon with different light constituent quark. As suggested by PYTHIA [50], the relative probability for various doubly heavy baryons is $\sigma_{\Xi_{Q Q^{\prime} u}}: \sigma_{\Xi_{Q Q^{\prime} d}}: \sigma_{\Xi_{Q Q^{\prime} s}}=10: 10: 3$. Then, for the produced $\Xi_{c c}$ events, one expects $43 \%$ to be $\Xi_{c c}^{++}, 43 \%$ to be $\Xi_{c c}^{+}$, and $14 \%$ to be $\Omega_{c c}^{+}$. The same situation occurs for the production of $\Xi_{b b}^{0}, \Xi_{b b}^{-}, \Omega_{b b}^{-}$, and $\Xi_{b c}^{+}, \Xi_{b c}^{0}, \Omega_{b c}^{0}$.

\section{Acknowledgments}

This work was supported in part by the Fundamental Research Funds for the Central Universities under Grant No.CQDXWL-2012-Z002 and by the Natural Science Foundation of China under Grant No.11275280.

Open Access. This article is distributed under the terms of the Creative Commons Attribution License (CC-BY 4.0), which permits any use, distribution and reproduction in any medium, provided the original author(s) and source are credited.

\section{References}

[1] A.F. Falk, M.E. Luke, M.J. Savage and M.B. Wise, Heavy quark fragmentation to baryons containing two heavy quarks, Phys. Rev. D 49 (1994) 555 [hep-ph/9305315] [INSPIRE]. 
[2] V.V. Kiselev, A.K. Likhoded and M.V. Shevlyagin, Double charmed baryon production at B factory, Phys. Lett. B 332 (1994) 411 [hep-ph/9408407] [INSPIRE].

[3] S.P. Baranov, On the production of doubly flavored baryons in $p p$, ep and $\gamma \gamma$ collisions, Phys. Rev. D 54 (1996) 3228 [inSPIRE].

[4] A.V. Berezhnoy, V.V. Kiselev, A.K. Likhoded and A.I. Onishchenko, Doubly charmed baryon production in hadronic experiments, Phys. Rev. D 57 (1998) 4385 [hep-ph/9710339] [INSPIRE].

[5] J.P. Ma and Z.G. Si, Factorization approach for inclusive production of doubly heavy baryon, Phys. Lett. B 568 (2003) 135 [hep-ph/0305079] [INSPIRE].

[6] S.-Y. Li, Z.-G. Si and Z.-J. Yang, Doubly heavy baryon production at $\gamma \gamma$ collider, Phys. Lett. B 648 (2007) 284 [hep-ph/0701212] [INSPIRE].

[7] C.-H. Chang, C.-F. Qiao, J.-X. Wang and X.-G. Wu, Estimate of the hadronic production of the doubly charmed baryon $\Xi_{c c}$ under GM-VFN scheme, Phys. Rev. D 73 (2006) 094022 [hep-ph/0601032] [INSPIRE].

[8] C.-H. Chang, J.-P. Ma, C.-F. Qiao and X.-G. Wu, Hadronic production of the doubly charmed baryon $\Xi_{c c}$ with intrinsic charm, J. Phys. G 34 (2007) 845 [hep-ph/0610205] [INSPIRE].

[9] C.-H. Chang, J.-X. Wang and X.-G. Wu, GENXICC: A generator for hadronic production of the double heavy baryons $\Xi_{c c}, \Xi_{b c}$ and $\Xi_{b b}$, Comput. Phys. Commun. 177 (2007) 467 [hep-ph/0702054] [INSPIRE].

[10] C.-H. Chang, J.-X. Wang and X.-G. Wu, GENXICC2.0: An Upgraded Version of the Generator for Hadronic Production of Double Heavy Baryons $\Xi_{c c}, \Xi_{b c}$ and $\Xi_{b b}$, Comput. Phys. Commun. 181 (2010) 1144 [arXiv:0910.4462] [INSPIRE].

[11] J.-W. Zhang, X.-G. Wu, T. Zhong, Y. Yu and Z.-Y. Fang, Hadronic Production of the Doubly Heavy Baryon $\Xi_{b c}$ at LHC, Phys. Rev. D 83 (2011) 034026 [arXiv:1101.1130] [INSPIRE].

[12] G. Chen, X.-G. Wu, J.-W. Zhang, H.-Y. Han and H.-B. Fu, Hadronic production of $\Xi_{c c}$ at a fixed-target experiment at the LHC, Phys. Rev. D 89 (2014) 074020 [arXiv:1401.6269] [INSPIRE].

[13] J. Jiang, X.-G. Wu, Q.-L. Liao, X.-C. Zheng and Z.-Y. Fang, Doubly Heavy Baryon Production at A High Luminosity $e^{+} e^{-}$Collider, Phys. Rev. D 86 (2012) 054021 [arXiv: 1208.3051] [INSPIRE].

[14] J. Jiang, X.-G. Wu, S.-M. Wang, J.-W. Zhang and Z.-Y. Fang, A Further Study on the Doubly Heavy Baryon Production around the $Z^{0}$ Peak at A High Luminosity $e^{+} e^{-}$Collider, Phys. Rev. D 87 (2013) 054027 [arXiv: 1302.0601] [INSPIRE].

[15] SELEX collaboration, M. Mattson et al., First observation of the doubly charmed baryon $\Xi_{c c}^{+}$, Phys. Rev. Lett. 89 (2002) 112001 [hep-ex/0208014] [INSPIRE].

[16] SELEX collaboration, A. Ocherashvili et al., Confirmation of the double charm baryon $\Xi_{c c}^{+}(3520)$ via its decay to $p D^{+} K^{-}$, Phys. Lett. B 628 (2005) 18 [hep-ex/0406033] [INSPIRE].

[17] V.V. Kiselev and A.K. Likhoded, Comment on 'First observation of doubly charmed baryon $\Xi_{c c}^{+}$, hep-ph/0208231 [INSPIRE].

[18] LHCb collaboration, Implications of LHCb measurements and future prospects, Eur. Phys. J. C 73 (2013) 2373 [arXiv:1208.3355] [INSPIRE]. 
[19] LHCb collaboration, Search for the doubly charmed baryon $\Xi_{c c}^{+}$, JHEP 12 (2013) 090 [arXiv: 1310.2538] [INSPIRE].

[20] BeLle collaboration, Y. Kato et al., Search for doubly charmed baryons and study of charmed strange baryons at Belle, Phys. Rev. D 89 (2014) 052003 [arXiv:1312.1026] [INSPIRE].

[21] G.T. Bodwin, E. Braaten and G.P. Lepage, Rigorous QCD analysis of inclusive annihilation and production of heavy quarkonium, Phys. Rev. D 51 (1995) 1125 [Erratum ibid. D 55 (1997) 5853] [hep-ph/9407339] [INSPIRE].

[22] C.-H. Chang, C. Driouichi, P. Eerola and X.G. Wu, BCVEGPY: An event generator for hadronic production of the $B_{c}$ meson, Comput. Phys. Commun. 159 (2004) 192 [hep-ph/0309120] [INSPIRE].

[23] E. Braaten, M. Kusunoki, Y. Jia and T. Mehen, $\Lambda_{c}^{+} / \Lambda_{c}^{-}$asymmetry in hadroproduction from heavy quark recombination, Phys. Rev. D 70 (2004) 054021 [hep-ph/0304280] [INSPIRE].

[24] W.K. Lai and A.K. Leibovich, $\Lambda_{c}^{+} / \Lambda_{c}^{-}$and $\Lambda_{b}^{0} / \bar{\Lambda}_{b}^{0}$ production asymmetry at the LHC from heavy quark recombination, arXiv:1410.2091 [INSPIRE].

[25] I.F. Ginzburg, G.L. Kotkin, V.G. Serbo and V.I. Telnov, Colliding $\gamma e$ and $\gamma \gamma$ Beams Based on the Single Pass Accelerators (of Vlepp Type), Nucl. Instrum. Meth. 205 (1983) 47 [INSPIRE].

[26] V.I. Telnov, Problems of Obtaining $\gamma \gamma$ and $\gamma \epsilon$ Colliding Beams at Linear Colliders, Nucl. Instrum. Meth. A 294 (1990) 72 [inSPIRE].

[27] C. Peterson, D. Schlatter, I. Schmitt and P.M. Zerwas, Scaling Violations in Inclusive $e^{+} e^{-}$ Annihilation Spectra, Phys. Rev. D 27 (1983) 105 [INSPIRE].

[28] V.G. Kartvelishvili, A.K. Likhoded and V.A. Petrov, On the Fragmentation Functions of Heavy Quarks Into Hadrons, Phys. Lett. B 78 (1978) 615 [InSPIRE].

[29] M.G. Bowler, $e^{+} e^{-}$Production of Heavy Quarks in the String Model, Z. Phys. C 11 (1981) 169 [InSPIRE].

[30] P.D.B. Collins and T.P. Spiller, The Fragmentation of Heavy Quarks, J. Phys. G 11 (1985) 1289 [inSPIRE].

[31] G. Colangelo and P. Nason, A Theoretical study of the $c$ and b fragmentation function from $e^{+} e^{-}$annihilation, Phys. Lett. B 285 (1992) 167 [InSPIRE].

[32] E. Braaten, K.-m. Cheung, S. Fleming and T.C. Yuan, Perturbative QCD fragmentation functions as a model for heavy quark fragmentation, Phys. Rev. D 51 (1995) 4819 [hep-ph/9409316] [INSPIRE].

[33] P. Masjuan, $\gamma * \gamma \rightarrow \pi^{0}$ transition form factor at low-energies from a model-independent approach, Phys. Rev. D 86 (2012) 094021 [arXiv: 1206. 2549] [INSPIRE].

[34] S. Fleming, A.K. Leibovich, T. Mehen and I.Z. Rothstein, Anomalous dimensions of the double parton fragmentation functions, Phys. Rev. D 87 (2013) 074022 [arXiv:1301.3822] [INSPIRE].

[35] Y.-Q. Ma, J.-W. Qiu and H. Zhang, Heavy quarkonium fragmentation functions from a heavy quark pair. I. S wave, Phys. Rev. D 89 (2014) 094029 [arXiv:1311.7078] [INSPIRE].

[36] Y.-Q. Ma, J.-W. Qiu and H. Zhang, Heavy quarkonium fragmentation functions from a heavy quark pair. II. P wave, Phys. Rev. D 89 (2014) 094030 [arXiv:1401.0524] [INSPIRE]. 
[37] Z.-B. Kang, Y.-Q. Ma, J.-W. Qiu and G. Sterman, Heavy Quarkonium Production at Collider Energies: Factorization and Evolution, Phys. Rev. D 90 (2014) 034006 [arXiv: 1401.0923] [INSPIRE].

[38] Y.-Q. Ma, J.-W. Qiu, G. Sterman and H. Zhang, Factorized power expansion for high-p heavy quarkonium production, Phys. Rev. Lett. 113 (2014) 142002 [arXiv:1407.0383] [INSPIRE].

[39] A.V. Berezhnoy, V.V. Kiselev, A.K. Likhoded and A.I. Onishchenko, Doubly charmed baryon production in hadronic experiments, Phys. Rev. D 57 (1998) 4385 [hep-ph/9710339] [INSPIRE].

[40] SLD collaboration, K. Abe et al., Measurement of the $b$ quark fragmentation function in $Z^{0}$ decays, Phys. Rev. D 65 (2002) 092006 [Erratum ibid. D 66 (2002) 079905] [hep-ex/0202031] [INSPIRE].

[41] DELPHI collaboration, J. Abdallah et al., A measurement of the branching fractions of the $b$ quark into charged and neutral b hadrons, Phys. Lett. B 576 (2003) 29 [hep-ex/0311005] [INSPIRE].

[42] SLD collaboration, K. Abe et al., Measurements of $R_{b}$ with impact parameters and displaced vertices, Phys. Rev. D 53 (1996) 1023 [InSPIRE].

[43] ALEPH collaboration, A. Heister et al., Study of the fragmentation of b quarks into B mesons at the $Z$ peak, Phys. Lett. B 512 (2001) 30 [hep-ex/0106051] [INSPIRE].

[44] OPAL collaboration, G. Abbiendi et al., Inclusive analysis of the $b$ quark fragmentation function in $Z$ decays at LEP, Eur. Phys. J. C 29 (2003) 463 [hep-ex/0210031] [INSPIRE].

[45] C. Yu-qi and W. Su-zhi, Production of four-quark states with double heavy quarks at LHC, Phys. Lett. B 705 (2011) 93 [arXiv:1101.4568] [InSPIRE].

[46] G. Chen, X.-G. Wu, H.-B. Fu, H.-Y. Han and Z. Sun, Photoproduction of the heavy quarkonium at the ILC, Phys. Rev. D 90 (2014) 034004 [arXiv: 1407.3650] [INSPIRE].

[47] A. Petrelli, M. Cacciari, M. Greco, F. Maltoni and M.L. Mangano, NLO production and decay of quarkonium, Nucl. Phys. B 514 (1998) 245 [hep-ph/9707223] [INSPIRE].

[48] S.-Q. Wang, X.-G. Wu, X.-C. Zheng, J.-M. Shen and Q.-L. Zhang, $J / \psi+\chi_{c J}$ Production at the B Factories under the Principle of Maximum Conformality,

Nucl. Phys. B 876 (2013) 731 [arXiv:1301.2992] [INSPIRE].

[49] X.-C. Zheng, X.-G. Wu, S.-Q. Wang, J.-M. Shen and Q.-L. Zhang, Reanalysis of the BFKL Pomeron at the next-to-leading logarithmic accuracy, JHEP 10 (2013) 117 [arXiv: 1308.2381] [INSPIRE].

[50] T. Sjöstrand, S. Mrenna and P.Z. Skands, PYTHIA 6.4 Physics and Manual, JHEP 05 (2006) 026 [hep-ph/0603175] [INSPIRE]. 